\title{
Entanglement dynamics at flat surfaces: investigations using multi-chain molecular dynamics and a single-chain slip-spring model
}

Article

Accepted Version

Kirk, J., Wang, Z. and Ilg, P. (2019) Entanglement dynamics at flat surfaces: investigations using multi-chain molecular dynamics and a single-chain slip-spring model. The Journal of Chemical Physics, 150 (9). 094906. ISSN 0021-9606 doi: https://doi.org/10.1063/1.5045301 Available at https://centaur.reading.ac.uk/82599/

It is advisable to refer to the publisher's version if you intend to cite from the work. See Guidance on citing.

To link to this article DOI: http://dx.doi.org/10.1063/1.5045301

Publisher: American Institute of Physics

All outputs in CentAUR are protected by Intellectual Property Rights law, including copyright law. Copyright and IPR is retained by the creators or other copyright holders. Terms and conditions for use of this material are defined in the End User Agreement. 


\section{CentAUR}

Central Archive at the University of Reading

Reading's research outputs online 


\title{
Entanglement dynamics at flat surfaces: investigations using multi-chain Molecular Dynamics and a single-chain slip-spring model
}

\author{
Jack Kirk, ${ }^{1}$ Zuowei Wang, ${ }^{1}$ and Patrick lg $^{1}$ \\ School of Mathematical, Physical and Computational Sciences, \\ University of Reading, Reading RG6 6AX, UK ${ }^{\mathrm{a})}$
}

(Dated: 4 March 2019)

The dynamics of an entangled polymer melt confined in a channel by parallel plates is investigated by Molecular Dynamics (MD) simulations of a detailed, multi-chain model. A Primitive Path Analysis predicts that the density of entanglements remains approximately constant throughout the gap and drops to lower values only in the immediate vicinity of the surface. Based on these observations, we propose a coarsegrained, single-chain slip-spring model with a uniform density of slip-spring anchors and slip-links. The slip-spring model is compared to the Kremer-Grest MD beadspring model via equilibrium correlation functions of chain orientations. Reasonably good agreement between the single-chain model and the detailed multi-chain model is obtained for chain relaxation dynamics, both away from the surface and for chains whose center of mass positions are at a distance from the surface that is less than the bulk chain radius of gyration, without introducing any additional model parameters. Our results suggest that there is no considerable drop in topological interactions for chains in the vicinity of a single flat surface. We infer from the slip-spring model that the experimental plateau modulus of a confined polymer melt may be different to a corresponding unconfined system even if there is no drop in topological interactions for the confined case.

a) p.ilg@reading.ac.uk 


\section{INTRODUCTION}

The dynamics of confined polymers is an interesting subject that is particularly important for the behavior of nanocomposite materials ${ }^{1,2}$, the phenomenon of surface slip in polymer melts $^{3,4}$, and additive manufacturing ${ }^{5}$. However the dynamics are difficult to characterise and predict. A key concept is the behavior of entanglements between different polymer molecules in the vicinity of a confining surface. There has been much interest in the changes in properties and density of entanglements for such systems ${ }^{6-12}$. Ever since the seminal work by Fetters et al. ${ }^{13}$, the success of the Lin-Noolandi conjecture ${ }^{14-16}$ has been cited in predictions of a large increase in the entanglement molecular weight for polymer melts under steric confinement ${ }^{17}$. Generally the justification for this prediction is the expectation that confined chains will be less inter-penetrating than their bulk counterparts. There is an experimental study which concluded that the prediction of a strong drop in entanglements near to the surface is correct even under weak confinement ${ }^{18}$.

An example of a relevant system is a polymer melt that is confined in a channel. Despite much investigation, there is no clear consensus regarding the magnitude and characteristic length of any depletion in the density profile of entanglements at a flat surface. For example, looking towards experiment, Bodiguel and Fretigny ${ }^{10}$ suggested that the density of entanglements at the surface might only deviate from the bulk value within approximately a tube diameter (entanglement spacing) from the surface, using polystyrene films. Weir et al. ${ }^{11}$ studied nano-composite systems comprising polymer melts filled with very high aspect ratio graphene oxide nano-particles. The authors suggest that the entanglement density could be reduced within roughly a chain radius of gyration from a surface.

Several studies using computer simulation have examined the system average 'entanglement length', $N_{e}$, of confined melts, found from Primitive Path Analysis (PPA ${ }^{19}$. In some cases an 'S-coil'"20 definition of entanglement length was employed ${ }^{7,12}$ that makes an assumption of Gaussian chain statistics. This assumption is usually considered appropriate in the bulk melt but appears questionable for confined systems. Alternative measures for the entanglement length that count the number of kinks in primitive paths which are generated using Kröger's $\mathrm{Z} 1$ algorithm ${ }^{21}$ have also been investigated under confinement ${ }^{22-24}$. One such measure, the 'modified S-kink' estimator, is:

$$
\mathscr{N}_{e}^{\text {topo }}(n)=\frac{n+1}{\langle Z\rangle}
$$


where $n$ is the number of bonds in a chain and $\langle Z\rangle$ is the mean number of kinks in a primitive path which separate straight line segments, such that:

$$
\lim _{n \rightarrow \infty} \mathscr{N}_{e}^{\text {topo }}(n)=N_{e}^{\text {topo }}
$$

Eqn 1 is sometimes referred to as a 'topological length'25. For a range of flexible polymer melts, eqn 1 underestimates the most widely used rheological definition of the entanglement length, $N_{e}^{\text {rheo }}$, by a factor of ${ }^{21} N_{e}^{\text {rheo }} / N_{e}^{\text {topo }} \approx 2$. A comparison with the S-coil measure, which agrees closely with the rheological definition, indicates that the primitive path, defined in this way, is not fully flexible. Everaers ${ }^{25}$ has argued that the ratio, $N_{e}^{\text {rheo }} / N_{e}^{\text {topo }}$, may be used to measure the functionality of the entanglement network using an analogy with phantom network theory. The observed ratio implies that entanglements are predominately binary events, roughly in agreement with a detailed investigation using Molecular Dynamics which nevertheless showed that a smaller but significant number of entanglements involve three chains $^{26}$. There is also evidence for the dominance of binary entanglements in simulations of star $^{27}$ and ring ${ }^{28}$ chain architectures. Here a relevant question is whether the binary picture is also valid for confined melts.

Although Molecular Dynamics can also allow the visualization of contacts between different chains that sometimes coincide with an intuitive picture of transient knots ('entanglements') ${ }^{26}$, it is not straightforward to understand how such topological interactions precisely influence chain dynamics and material properties ${ }^{27}$. It has already been pointed out that the relation between the plateau modulus and entanglement length may change under confinement ${ }^{22}$, and we will further consider this question in section 3. Even if topological interactions are binary, the motion of a given chain will depend more generally on the collective motion of chains in the surrounding environment that the given chain may not be directly in contact with $^{14}$. It then becomes unclear exactly how an entanglement can be most meaningfully defined, particularly when comparing chains in different environments. In our case, we would like to compare entanglements in chains at different positions relative to a surface. We would also like to stress that it is not a priori known how any entanglement measure found from microscopic simulation should correspond to the number of topological constraints (or fields) used in a theoretical coarse-grained model (e.g. tube or slip-spring models). For bulk melts, the relationships between PPA generated entanglement measures and the parameters controlling entanglements in theoretical models have been tested and are 
quite well understood ${ }^{19,21,25,29,30}$. In inhomogeneous systems comprising linear homopolymers the correspondence is not known since up till now no direct quantitative comparison between microscopic and coarse-grained models of such a system has been made for dynamic observables. For a mapping between microscopic and coarse-grained models of a diblock copolymer system see Ramírez-Hernández et al. ${ }^{31}$. Furthermore it is not obvious how the concepts of topological constraints ('entanglements') that are well defined in e.g. bulk slipspring models (slip-springs) or tube models (tube persistence length) may be extended to an inhomogeneous confined system.

Simply put, we must ask the following general question:

Is an entanglement near a surface similar to an entanglement in the bulk?

We focus on the relatively simple case of a polymer melt of linear and flexible chains that is weakly confined in a channel bounded by two parallel surfaces. The channel width is large compared to the size of a chain. To consider the above question for this case our approach is to generalize a theoretical bulk model with well defined topological fields to a confined system. Topological interactions are not an emergent phenomena within this empirical single-chain model, but are explicitly introduced via slip-springs. It is therefore more straightforward to investigate how the topological interactions affect chain dynamics near the surface within this model compared to detailed Molecular Dynamics where the definition of an entanglement is unclear. The single-chain slip-spring model is also vastly less computationally expensive than a corresponding MD simulation.

We compare the predictions of the single-chain slip-spring model with well defined topological constraints to a much more detailed many-chain molecular model. Quantitative comparison with the detailed MD model tests whether such the generalized slip-spring model can accurately predict dynamical observables, and if so, for which choice of density profile of topologically confining fields (slip-springs) across the inhomogeneous confined system.

Since confinement influences chain statistics and dynamics irrespective of whether topological interactions also exist, it is important that the slip-spring model also incorporates these effects. A detailed comparison of a simplified version of the model without slip-springs, to MD, has already been made ${ }^{32}$. This non-entangled 'modified-Rouse' model agrees reason- 
ably well with the behavior of sufficiently short Kremer-Grest (KG MD) model chains near the surface. Large deviations from the modified-Rouse model predictions, which emerge using longer KG MD model ${ }^{33}$ chains, are expected to be due to emergent topological interactions. This means that when we decorate the non-entangled modified-Rouse model with slip-springs, the degree of agreement of the resulting dynamics of the model with the entangled KG MD chains should give an indication of how well the slip-springs model the emergent effects of topological interactions at the surface.

It is necessary to prescribe a density profile of slip-links and slip-spring anchors in the channel. Simulation has also been employed to investigate entanglement depletion. Since primitive path kinks have a well defined spatial position, a kink density may be calculated as a description for an 'entanglement point' density profile. This has been carried out by Sussman ${ }^{24}$ for the case of free standing films using two different channel widths: $L_{c} \approx \sqrt{\left\langle R_{e}{ }^{2}\right\rangle_{\text {bulk }}}$ and $L_{c} \approx \sqrt{\left\langle R_{e}{ }^{2}\right\rangle_{\text {bulk }}} / 3$, for very long chains ( $n=2000$ bonds), where $\left\langle R_{e}{ }^{2}\right\rangle_{\text {bulk }}$ is the mean square end-to-end distance of chains in the bulk. In both cases, a very narrow depletion layer of kinks of width $\approx 4 \sigma$ was observed, where $\sigma$ is the Lennard-Jones length that is characteristic of a monomer size. A small maximum in the kink density profile at the border of the depletion layer was also observed. This observation is not well understood. Note that although the MD model of Sussman used a similar bead density to us, very stiff harmonic bonded potentials were used instead of the (Finitely Extensible Nonlinear Elastic) FENE springs of the standard KG MD model. We think that this should have only a small influence on chain statistics however.

It has been noted ${ }^{25}$ that for bulk systems the number of slip-springs per chain used in the slip-spring model to fit dynamical data of Molecular Dynamics simulations of KG MD model chains ${ }^{29,30}$ is very similar to the number of kinks per chain found using PPA. It is then tempting to heuristically associate a kink in a primitive path with a slip-spring in the slip-spring model. By associating kinks with slip-springs, as an initial working assumption, the relevant quantity that we would like to extract from MD is the kink density profile at the surface.

This paper is organized as follows: in section II we perform a PPA using the $Z 1$ algorithm, adapted for channel confinement, on a KG MD model of a melt confined in a channel by two parallel flat reflective surfaces. We generate a kink density profile as a function of 
distance to a surface analogous to the free film case carried out by Sussman. The kink density profile motivates the study of a simple single-chain slip-spring model under parallel plate confinement that assumes a uniform density of slip-links and anchors across the channel. Section III presents the slip-spring model. Finally, in section IV we compare the slip-spring model with the KG MD model using dynamical correlation functions of chains at equilibrium. Conclusions are made in section V.

\section{MOLECULAR DYNAMICS MODEL}

We use the same confined KG MD model and parameters for flexible chains as in a previous paper ${ }^{32}$, which is very similar to other models in the literature ${ }^{34,35}$. There are two parallel reflective surfaces bounding the fluid in one dimension so that the channel is symmetric, and periodic boundaries in the other two dimensions. The choice of flat reflecting surfaces is made for simplicity. Using simulations of melts comprising short chains we have verified that, for all auto-chain correlation function observables considered in this paper, this choice leads to no difference with respect to a surface formed by a rough lattice wall $^{36}$. It was however found ${ }^{36}$ that the choice of surface structure is very important for global observables such as the channel average stress correlation function ${ }^{35,36}$ and crosschain orientation correlation functions ${ }^{36,37}$.

We investigate a monodisperse melt comprising $N_{c h}=357$ chains, each with $n=256$ bonds. A Langevin thermostat with friction constant $\xi_{\mathrm{MD}}=0.5 m_{b} / \tau_{\mathrm{LJ}}$ was used, where $m_{b}$ is the bead mass and $\tau_{\mathrm{LJ}}=\sqrt{\sigma^{2} m_{b} / \epsilon}$ is the shortest natural time unit of the simulation ('LennardJones time'); $\epsilon$ and $\sigma$ are the Lennard-Jones interaction energy and length respectively. The temperature was set to $T=\epsilon$. The periodic box dimensions parallel to the surface were set as $L_{z}, L_{x} \approx 2 \sqrt{\left\langle R_{e}{ }^{2}\right\rangle_{\text {bulk }}}$. We choose a channel width normal to the confining surfaces of $L_{c} \approx 3.4 \sqrt{\left\langle R_{e}{ }^{2}\right\rangle_{\text {bulk }}}$. This choice ensures that there is a center channel region with width $0.5 \sqrt{\left\langle R_{e}{ }^{2}\right\rangle_{\text {bulk }}}$ that only comprises chains whose mean square end-to-end vectors match those of bulk chains ${ }^{32}$. For the chain length investigated, $n=256, \sqrt{\left\langle R_{e}{ }^{2}\right\rangle_{\text {bulk }}} \approx 21 \sigma$. The monomer density in the channel center matches the standard bulk density of $\rho=0.85 \sigma^{-3}$ for a Kremer-Grest melt. 


\section{Equilibration procedure}

In addition to the confined channel system preparation for shorter chains described previously $^{32}$, additional equilibration steps for the entangled system with $n=256$ are necessary. We here follow the procedure of Cao and Likhtman ${ }^{38}$ by first preparing the system using soft non-bonded and harmonic bonded potentials which allow bond-crossing events. This set of soft interaction potentials has been termed 'soft MD'39. We have previously demonstrated that in the presence of a flat reflective surface the soft MD model has very similar static properties to $\mathrm{KG} \mathrm{MD}^{32}$. Simulations using the soft MD model ran for several Rouse times. Following this the potentials were switched to the KG MD model using a 'push-off' over the first Lennard-Jones time, $\tau_{\mathrm{LJ}}$. The system then ran for several longest KG MD system relaxation times, $\tau_{\mathrm{F}}$, (defined from the longest Maxwell mode of a spectrum fitted to the surface parallel end-to-end vector correlation function (where in $\tau_{\mathrm{F}}$ ' $\mathrm{F}$ ' denotes that all chains have two free ends in this system)) before any data presented here was collected. For $n=256$ we find that $\tau_{\mathrm{F}} \approx 2.5 \times 10^{5} \tau_{\mathrm{LJ}}$. Several independent runs were carried out and the results were obtained by an ensemble average. Checking that the data recorded in consecutive runs was consistent acted as an additional check that the system was properly equilibrated. For every observable, error bars are calculated based on the assumption that recorded data generated from conformations which are separated by $2.4 \tau_{\mathrm{F}}$ are uncorrelated. The error bars represent confidence intervals of one standard deviation.

\section{A Primitive Path Analysis of a confined polymer melt}

We use the Z1 algorithm of Kröger ${ }^{21}$ in order to determine the spatial locations of kinks in the primitive paths of polymer chains. Figure 1 plots the kink density as a function of distance from the nearest wall, normalized by the mean kink density in the channel center. There is a depletion layer of kinks until approximately $y=2 \sigma$ from the surface, immediately followed by a moderate excess of kinks before the bulk behavior is reached at approximately $y=6 \sigma$ from the surface.

When we map to the slip-spring model in section III the mean slip-spring ('virtual') bond length is $b_{v} \approx 2.9 \sigma$ when mapped to the KG MD model length scale. Unlike kinks, slipsprings act as confining fields rather that network points. A model using a small number 


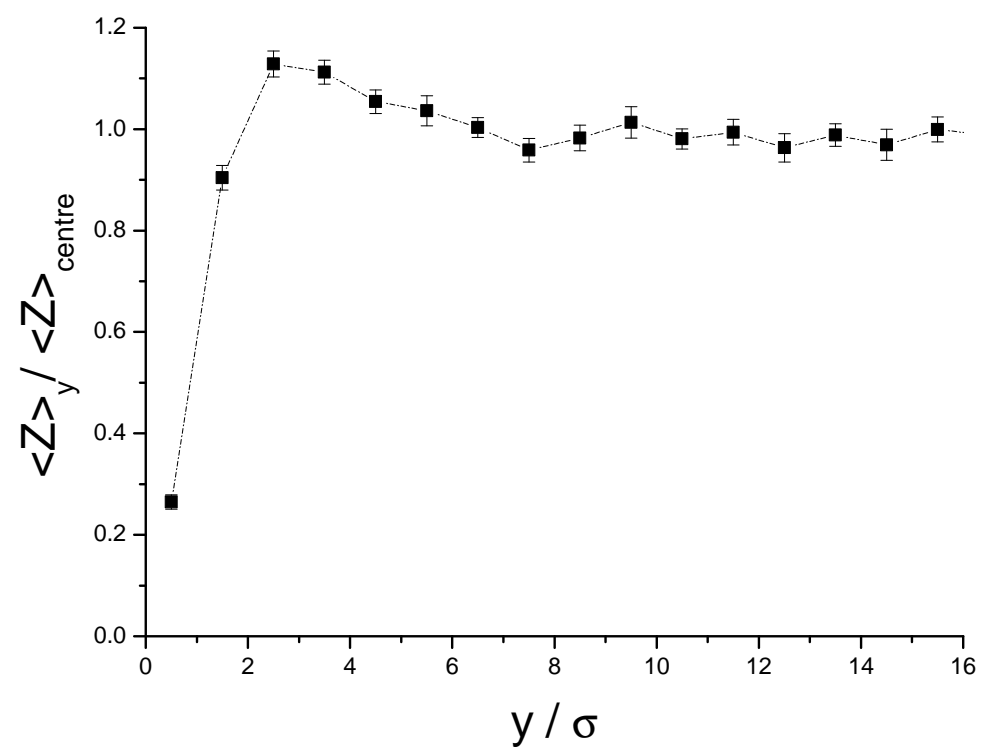

FIG. 1: Kink density profiles as a function of distance from the kink to the nearest surface, normalized by the center channel density. Kinks are obtained via the Z1 algorithm ${ }^{21}$ applied to the KG MD melt using chains comprising $n=256$ bonds.

of stiff slip-springs can produce similar results to a model using a larger number of softer slip-springs. However the best fit to bulk MD data is found using our chosen parameters ${ }^{29,30}$. As a result it may not be very meaningful to try to fit slip-link or anchor density profiles precisely to the kink density profile on length-scales smaller than approximately $b_{v}$.

Taking into account the region with increased kink density, the mean density in the surface region (of width $\approx 6 \sigma \approx 2 b_{v}$ ) is only a little less than in the channel center (approximately $10 \%$ less). Primitive paths may also be found by 'non-destructive' methods such as the Isoconfigurational Ensemble (ICE) method ${ }^{6}$. ICE defines a primitive path, that is consistent with the classical picture of tube models, by averaging chain conformations over a time interval of order the equilibration time, $\tau_{e}$ : the time at which topological contraints become important for dynamic correlations so that a 'phantom chain' description such as the modified-Rouse model becomes insufficient. Several simulations with identical initial spatial configurations but different initial bead velocities are averaged over. Alternative definitions of 'entanglement points' have been proposed using $\mathrm{ICE}^{40}$, and a comparison between the primitive paths that are generated using either a Z1 type algorithm or ICE has been made, demonstrating good qualitative agreement between the two methods ${ }^{6}$. The particular form of a density profile for 'entanglement points' is expected to depend to some 
degree on their particular definition. This is another reason for why we consider that the mean kink density in the surface region is the most important quantity to extract with regard to initially informing an appropriate slip-link density profile. Further analysis using a range of chain-lengths is necessary in order to establish a good understanding for the scaling of the size of any 'entanglement point' surface depletion, preferably using more than one definition of 'entanglement point'. It has been postulated ${ }^{41}$, that the drop in kinks at the surface could be due to the weak nematic interaction for segments close to the surface ${ }^{32}$, but this possibility has not been tested. We also note that Figure 1 is qualitatively similar to the density profile of the chain center of mass near the surface (see Figure 4 of the supplementary material). Figure 1 shows that the drop in kinks in the surface region of size $\approx 2 b_{v}$ is small for the chain length studied. However we must note that although we do not have a surface density profile of 'entanglement points' that is generated using a 'non-destructive' method, the ICE methodology applied to ring polymers concluded that the tube diameter is larger than in the bulk within two or three bulk tube diameters from the surface of a free-standing film by a maximum factor of $15-20 \%$. Finally we note that the Z1 algorithm ignores self entanglements. A previous PPA investigation concluded that the number of self-entanglements is negligible in the bulk ${ }^{42}$. In the future it would be useful to also examine the importance of self-entanglements at the surface.

In the following section we present an extension of the original single-chain slip-spring model, developed by Likhtman ${ }^{43}$, to include confinement effects. We investigate the effect that surface proximity has on chain dynamics under the assumption that there is a uniform

density of slip-links and anchors at all points in the channel. Since the average kink density near the surface is only $\approx 10 \%$ less than in the channel center, this assumption appears to be a reasonable initial guess.

\section{A SLIP-SPRING MODEL WITH PARALLEL PLATE CONFINEMENT}

The single-chain slip-spring model presented here aims to describe entangled chains in a polymer melt at flat and completely non-adsorbing surfaces. It is an adaptation of the original model by Likhtman ${ }^{43}$ with no additional parameters, and a natural extension of a non-entangled modified-Rouse model for surface chain dynamics in weak confinement ${ }^{32}$. 


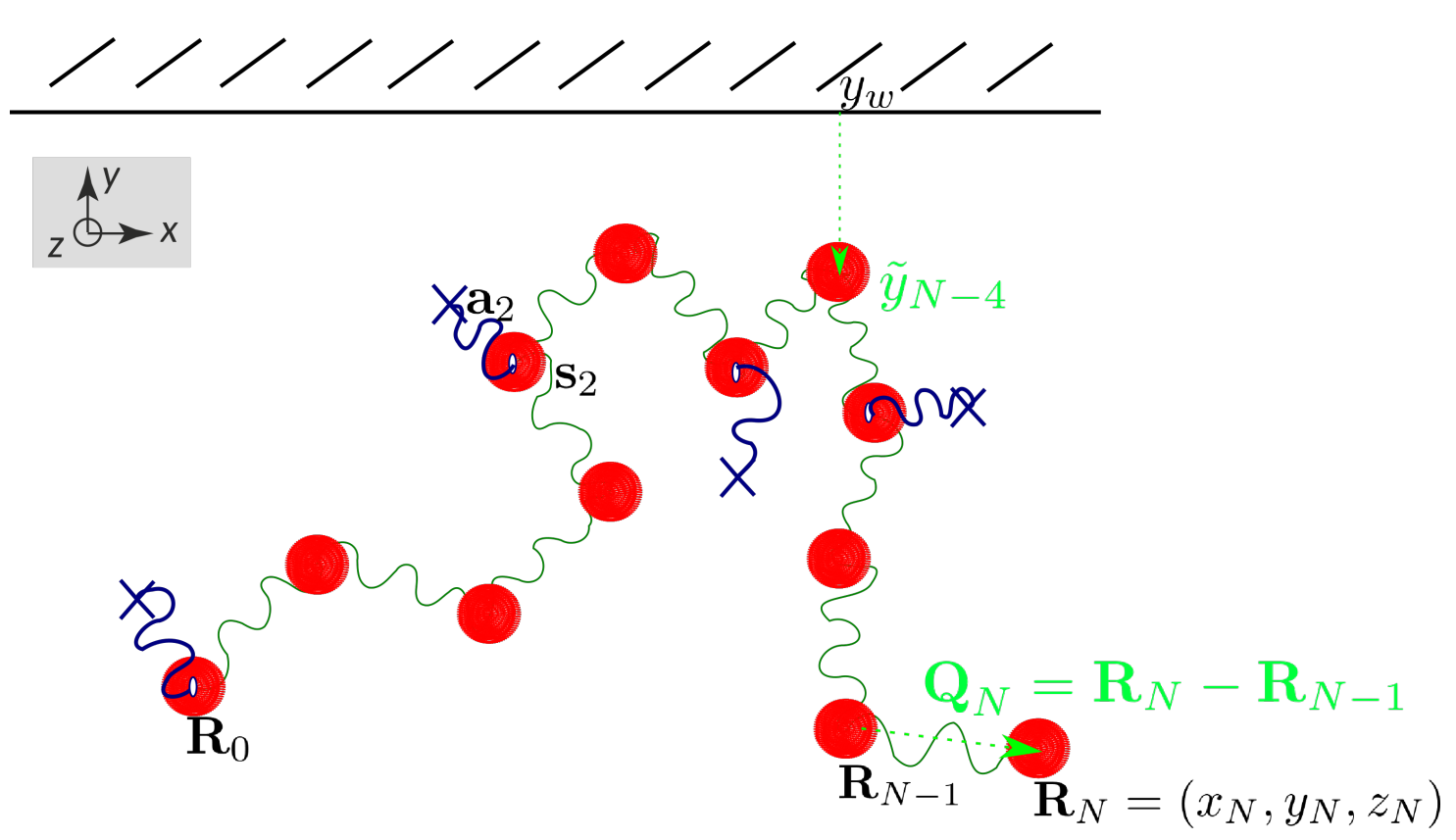

FIG. 2: Schematic illustration of a single chain comprising $N=11$ real springs (represented by green wavy curves) and $Z=4$ virtual springs (blue wavy curves), within the confined slip-spring model. The positions, $\boldsymbol{R}_{0}$ and $\boldsymbol{R}_{N}$, of the first and last beads, are indicated. The final bond vector $\boldsymbol{Q}_{N}=\boldsymbol{R}_{N}-\boldsymbol{R}_{N-1}$ is also indicated. The virtual springs

(slip-springs) are connected to real chain beads via slip-links at positions, $\boldsymbol{s}_{j}$. The slip-springs are fixed to anchoring points at positions $\boldsymbol{a}_{j}$, as represented via crosses. The positions of the anchor and slip-link of the second slip-spring are indicated in the illustration. Positions relative to the nearest wall (located at $y_{w}$ ) are indicated by tildes. For example $\tilde{y}_{N-4}$ indicates the position of the $N-4$ th bead relative to the nearest wall,

$$
\tilde{y}_{N-4}=y_{N-4}-y_{w} .
$$

In the same spirit as the original work ${ }^{43}$ where slip-links are added to the standard Rouse model to capture entanglement effects, we here add slip-links to the modified-Rouse mode ${ }^{32}$ to include entanglement and confinement effects. The slip-links are connected to anchors via a 'spring' potential that is modified with respect to the Hookean spring used in the original slip-spring model. A schematic illustration of a single chain within the model, indicating all degrees of freedom, is given by figure 2 . The total chain potential in the presence of a single wall at position $y_{w} \hat{\boldsymbol{e}}_{\perp}$, including the sets of interactions due to $N$ real 'springs' connecting the modified-Rouse beads and $Z$ virtual 'springs' acting between slip-links at positions $\boldsymbol{s}_{j}$ 
and anchors at positions $\boldsymbol{a}_{j}$, is given by

$$
\begin{aligned}
U_{S S} & =U_{N}\left(\left\{\boldsymbol{R}^{N+1}\right\}, y_{w}\right)+U_{Z}\left(\left\{\boldsymbol{s}^{Z}\right\},\left\{\boldsymbol{a}^{Z}\right\}, y_{w}\right) \\
& =\sum_{i=1}^{N}\left(\frac{k}{2} \boldsymbol{Q}_{i}{ }^{2}+A\left(y_{i}, y_{i-1}, y_{w}\right)\right) \\
& +\sum_{j=1}^{Z}\left(\frac{k}{2 N_{s}}\left(\boldsymbol{s}_{j}-\boldsymbol{a}_{j}\right)^{2}+A\left(s_{j, y}, a_{j, y}, y_{w}\right)\right)
\end{aligned}
$$

where $\boldsymbol{R}_{i}$ and $\boldsymbol{Q}_{i}=\boldsymbol{R}_{i}-\boldsymbol{R}_{i-1}$ are the real chain bead and bond vectors respectively, $k=T / \sigma_{1}{ }^{2}, \sigma_{1}{ }^{2}=b^{2} / 3$, and $b^{2}$ is equal to the mean square bond length far from the surface. Temperature, $T$, is written in units of the Boltzmann constant, $k_{b}$. Surface normal positions are defined as $a_{j, y}=\boldsymbol{a}_{j} \cdot \hat{\boldsymbol{e}}_{\perp}$ and $y_{i}=\boldsymbol{R}_{i} \cdot \hat{\boldsymbol{e}}_{\perp} \cdot N_{s}$ is the "number of monomers per virtual spring' 43 which controls the mean square virtual spring length far from the surface, $b_{v}{ }^{2}$, via the relation: $b_{v}{ }^{2}=N_{s} b^{2}$. The first part of eqn 3 is the standard modified-Rouse potential previously investigated $^{32}$. The modified-Rouse potential leads to reflected-Gaussian bond statistics and a uniform bead density at all points in the channel, such that the fluid is considered incompressible. To this is added the virtual-spring potential, $U_{Z}\left(\left\{\boldsymbol{s}^{Z}\right\},\left\{\boldsymbol{a}^{Z}\right\}, y_{w}\right)$, which differs from the original slip-spring potential via the additional surface dependent term

$$
A\left(s_{j, y}, a_{j, y}, y_{w}\right)=-T \log \left(1+\exp \left(-\frac{2 \tilde{s}_{j, y} \tilde{a}_{j, y}}{N_{s} \sigma_{1}{ }^{2}}\right)\right)
$$

where tildes indicate relative wall positions, e.g. $\tilde{s}_{j, y}=s_{j, y}-y_{w}$.

In the surface parallel direction the probability distribution of the complete set of real and virtual spring orientations is identical to the original slip-spring model. In the wall normal direction the probability distribution becomes

$$
\begin{aligned}
& \Psi\left(\left\{y^{N+1}\right\},\left\{s_{y}^{Z}\right\},\left\{a_{y}^{Z}\right\}\right) \propto \\
& \exp \left(-\sum_{i=1}^{N}\left(\frac{\left(y_{i}-y_{i-1}\right)^{2}}{2 \sigma_{1}{ }^{2}}+\frac{\left(-\tilde{y}_{i}-\tilde{y}_{i-1}\right)^{2}}{2 \sigma_{1}{ }^{2}}\right)\right) \times \\
& \exp \left(-\sum_{j=1}^{Z}\left(\frac{\left(s_{j, y}-a_{j, y}\right)^{2}}{2 N_{s} \sigma_{1}{ }^{2}}+\frac{\left(-\tilde{s}_{j, y}-\tilde{a}_{j, y}\right)^{2}}{2 N_{s} \sigma_{1}{ }^{2}}\right)\right)
\end{aligned}
$$

The probability weight corresponding to the set of slip-spring orientations is proportional to the second exponential in eqn 5, which is in the form of a probability weight of a reflected random walk from anchor position $a_{j, y}$ to slip-link position $s_{j, y}$. Analogous to Likhtman's original slip-spring model ${ }^{43}$, integrating over all possible anchor positions in the channel 


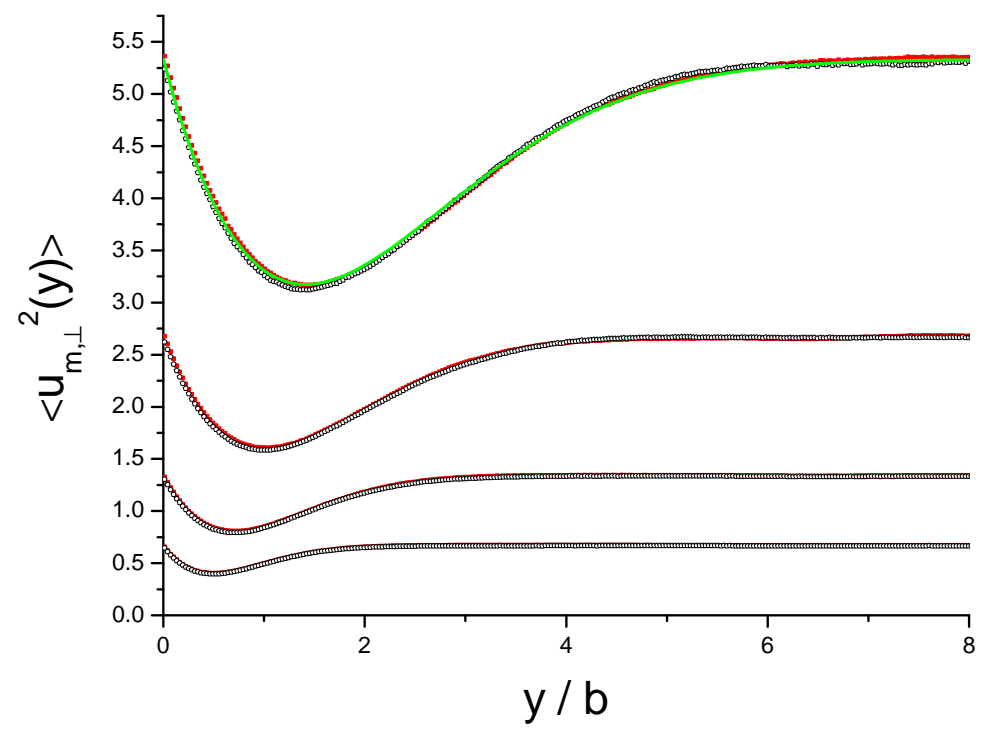

FIG. 3: Surface normal mean square end-to-end distances of segments, using segments consisting of $m=2,4,8$, and 16 bonds, as a function of segment start bead position with respect to a surface, $y$. Black open pentagons: modified-Rouse model (no slip-springs); red squares: slip-spring model using $N_{e}^{S S}=4$ and $N_{s}=0.5$; green line: analytic solution ${ }^{44}$ for $m=16$. Both models, with and without slip-springs, use chains with $N=32$ bonds.

leads to a probability distribution for the complete set of real chain beads that is unchanged with respect to the model without slip-links, since the partition function of a reflected random walk inside a channel is conserved irrespective of the walk start position. Figure 3 verifies that the surface normal mean square end-to-end distances of segments comprising $m$ bonds, $\left\langle u_{m, \perp}{ }^{2}\right\rangle$, match the model without slip-springs, at all points in the channel. The virtual-spring potential, $U_{Z}\left(\left\{\boldsymbol{s}^{Z}\right\},\left\{\boldsymbol{a}^{Z}\right\}, y_{w}\right)$, is a simple and natural choice that conserves the static behavior of the modified-Rouse model. If the additional term, eqn 4 , is neglected, the asymmetry of slip-spring orientations near the surface changes the distribution of real chain orientations. For an alternative approach that counteracts such an asymmetry, as applied to a multi-chain slip-spring model, see Masnada et al. ${ }^{45}$.

\section{Model implementation}

In order to create the initial configuration of the modified slip-spring model, the first bead of each chain is placed with wall normal position inside the channel according to a uniform distribution. Then the chains are grown by generating bond vectors according to a 
Gaussian distribution with zero mean and variance $b^{2}$. If a generated bead is placed outside of the channel then its position is reflected in the surface plane that it has crossed. This procedure results in a set of chain orientations sampling the distribution $\Psi$, eqn 5 .

Following the generation of the set of bead positions, a bead is selected from all beads in the channel with equal probability. If the selected bead is currently unoccupied then a sliplink is placed on it. The corresponding slip-spring anchor position is selected by making a displacement relative to the position of the slip-link according to a Gaussian distribution with zero mean and variance $N_{s} b^{2}$. If the anchor is outside of the channel then this position is reflected in the surface plane that it crossed. This leaves a set of anchor and slip-link positions sampling the distribution $\Psi$, eqn 5 . This system preparation is completed when there are $\operatorname{round}\left(N_{c h} N / N_{e}^{S S}\right)$ slip-springs in total in the channel, where $N_{c h}$ is the number of chains in the slip-spring simulation, and $N_{e}^{S S}$ is the mean number of bonds separating slip-springs. By construction the density of slip-links and anchors is uniform across the channel since the bead density is also uniform.

Slip-links may only exist at the position of chain beads. Slip-link dynamics are given by discrete moves according to a Metropolis-Hastings algorithm ${ }^{39}$ satisfying detailed balance. Slip-link jump attempts are made only to adjacent beads with equal probability: $\Delta i=$ \pm 1 , although in principle it would be possible to choose $|\Delta i| \geq 1$, where $\Delta i$ is an integer specifying the change in bead index following a slip-link jump. The acceptance probability of the jump attempt is given by

$$
P_{\text {accept }}= \begin{cases}1, & \Delta U_{S S} \leq 0 \\ \exp \left(-\Delta U_{S S} / T\right), & \Delta U_{S S}>0\end{cases}
$$

where $\Delta U_{S S}$ is the change in the chain potential, eqn 3, due to the Monte-Carlo move.

A slip-spring does not contribute to the system potential once it has left a chain end bead. This means that a slip-spring occupying an end-bead has a 50\% chance of being deleted every jump attempt. When a slip-link is deleted from a chain end, a new end-bead is selected from amongst all the chain end-beads in the channel with equal probability irrespective of the bead's position in the channel. If the selected end-bead is unoccupied a new slip-link is created on it. If the selected end-bead is occupied then the process is repeated by selecting another end-bead. The total number of slip-springs in the channel is then fixed for all time at round $\left(N_{c h} N / N_{e}^{S S}\right)$. 


\section{Parameters of the slip-spring model}

We use a slip-spring model comprising $N=26$ bonds to fit observables of the KG MD system comprising chains of $n=256$ bonds so that the mapping ratio is $n / N \approx 9.85$. We use the parameter values $N_{s}=0.5$ and $N_{e}^{S S}=4$ following earlier work ${ }^{30}$. A finer mapping may be used to get a better fit of observables at early time ${ }^{29}$. However this would be computationally expensive and unnecessary since we are interested in the dynamics at $t>\tau_{e}$. It is found that fine graining the slip-spring model by increasing proportionally $N, N_{s}$, and $N_{e}^{S S}$, makes very little difference to the dynamics at $t \gtrsim \tau_{e}$ for all observables investigated. For an investigation of the segmental dynamics at $t<\tau_{e}$, and details of the model dynamics for the real chain beads, see Kirk and $\operatorname{Ilg}^{32}$.

The channel width is chosen to be identical in units of $\sqrt{\left\langle R_{e}{ }^{2}\right\rangle_{\text {bulk }}}$ to the KG MD system $\left(L_{c} \approx 3.4 \sqrt{\left\langle R_{e}^{2}\right\rangle_{\text {bulk }}}\right)$. The time-step used is $\Delta t=0.01 \tau_{0}$ where

$$
\tau_{0}=\xi b^{2} / T
$$

refers to the time unit used by Zhu et al. ${ }^{46}$. For bulk chains it is already known ${ }^{30}$ that the slip-spring model time unit mapped from the KG MD model is $\tau_{0}=3370 \tau_{\mathrm{LJ}}$ using the chosen parameters, which is very similar ${ }^{30}$ to $\tau_{e}$. Following $\mathrm{Zhu}^{47}$ the frequency of slip-link jump attempts used is $f^{S S}=100 \tau_{0}^{-1}$ - i.e. a jump attempt every computational time-step interval.

\section{Constraint release}

Slip-links model the effect of constraints on the selected chain resulting from all the other chains in the melt. Since the other chains relax on the same time scale as the selected chain, the resulting constraints get destroyed and reorganized also on this time scale. This effect is called constraint release and can be implemented into the model by pairing up slip-links. If slip-links are paired then when one slip-link is deleted from a chain end a corresponding paired slip-link is also deleted and a move is attempted to regenerate the paired slip-link on any bead of any chain in the channel with equal probability. In a single-chain model 


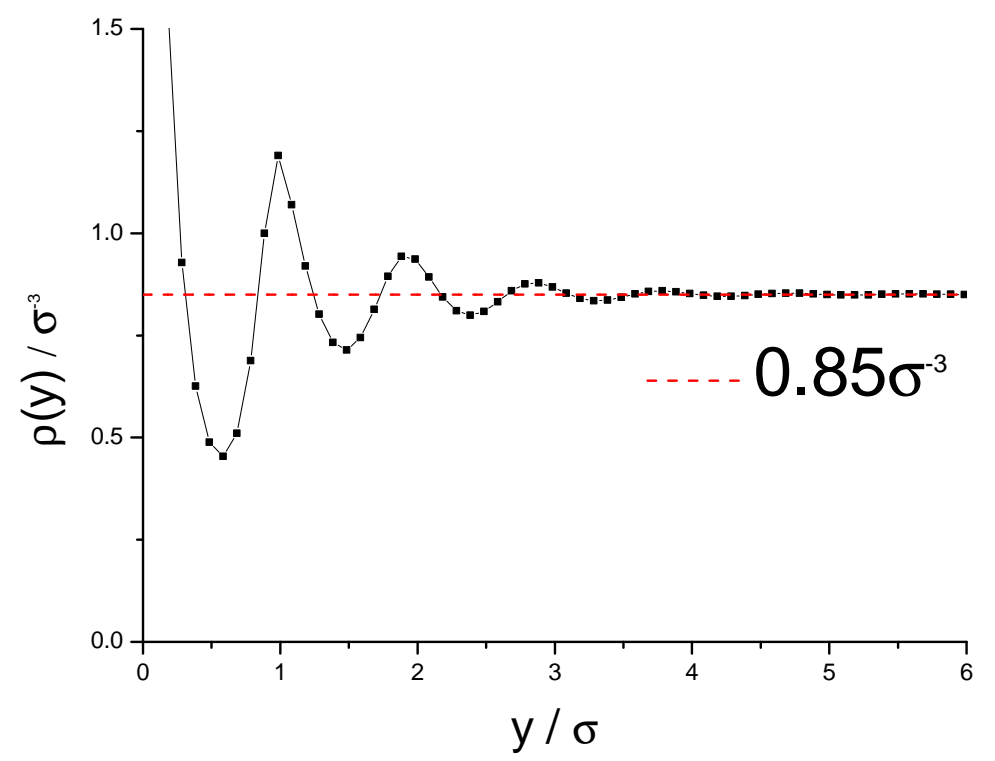

FIG. 4: Bead density profile near a flat reflective surface using the KG MD model with $n=256$ bonds per chain.

it only matters that the distribution of constraint release events is accurate. We observe that the distribution of slip-spring lifetimes is approximately independent of the channel position of the slip-spring when constraint release is switched off. In view of the simplicity of the resulting model, we follow the original approach of randomly pairing slip-links without consideration of the existence of confining surfaces. In the supplementary material the small differences in the slip-spring lifetime distributions as a function of the slip-spring position in the channel are investigated in detail using constraint release switched off.

\section{Density profile}

Figure 4 plots the bead density profile near a reflective surface using the KG MD model. The density oscillates near the surface but the mean density is very similar to the bulk value of $0.85 \sigma^{-3}$. Similar behavior has been observed using more realistic simulations of a polymer melt in contact with structured flat surfaces. For example, Daoulas et al. ${ }^{48}$ studied a united atom model of monodisperse polyethylene (PE) melts in contact with a graphite surface, spanning a decade of molecular weights up to chains consisting of 400 carbon atoms (the crossover to entanglement dominated dynamics occurs at around 156 Methyl groups $\left.\left(C_{156}\right)^{49}\right)$. Lee et al..$^{50}$ studied an all atom simulation of a polystyrene melt 
in contact with a graphite surface. In both cases no notable depletion in mass density was observed at the surfaces. In experiment, larger depletion layers, that are the size of a styrene monomer ${ }^{51}$, or greater ${ }^{52}$, have been found using polystyrene melts in contact with different surfaces. Theoretical models have been proposed to describe the density profile and conformations in the interfacial region where the polymer density is small but nonzero. For simplicity we assume a constant bead density for the modified slip-spring model proposed here. Discounting the oscillations in mass density in figure 4, this assumption is consistent with our particular MD model. Predictions of a drop in entanglements at the surface have been made without considering monomer depletion effects ${ }^{17}$, so that the simpler situation that we consider has at the very least considerable theoretical significance. When the interfacial width is found to be notable, this effect could be modeled ${ }^{53}$, as for example it has been in the detailed multi-chain slip-spring model of Ramírez-Hernández et al. ${ }^{54}$.

\section{CHAIN DYNAMICS: COMPARISON TO THE KG MD MODEL}

Chains are categorized into channel regions of wall normal width $L_{c} / 12 \approx 0.28 \sqrt{\left\langle R_{e}{ }^{2}\right\rangle_{\text {bulk }}}$ at $t^{\prime}=0$, depending on the distance, $y_{\mathrm{cm}}$, from the chain center of mass to the nearest wall at that time. For example, chains in the $j=0$ region closest to the surface satisfy $y_{\mathrm{cm}}<$ $0.28 \sqrt{\left\langle R_{e}^{2}\right\rangle_{\text {bulk }}}$ at $t^{\prime}=0$, whereas chains in the $j=1$ region satisfy $0.28 \sqrt{\left\langle R_{e}^{2}\right\rangle_{\text {bulk }}}<y_{\mathrm{cm}}<$ $0.56 \sqrt{\left\langle R_{e}^{2}\right\rangle_{\text {bulk }}}$. For ideal bulk chains the mean square radius of gyration satisfies ${ }^{55}\left\langle R_{g}{ }^{2}\right\rangle=$ $\left\langle R_{e}{ }^{2}\right\rangle / 6$. This means that the chains located in the two regions closest to the surface may be expected to be less entangled than bulk chains according to previous work ${ }^{11,17}$, since such chains are located within approximately one radius of gyration from the surface. Since the channel is symmetric we improve statistics by averaging opposing regions.

We investigate time correlation functions of form

$$
\begin{aligned}
F_{j}(t) & =\left\langle\frac{\sum_{i=1}^{N_{c h}} \Theta\left(y_{c m}^{i}(0)-\mathcal{Y}_{j}\right) \Theta\left(\mathcal{Y}_{j+1}-y_{c m}^{i}(0)\right) \int_{0}^{t_{\max }-t} f^{i}\left(t^{\prime}+t\right) f^{i}\left(t^{\prime}\right) d t^{\prime}}{\sum_{i=1}^{N_{c h}} \Theta\left(y_{c m}^{i}(0)-\mathcal{Y}_{j}\right) \Theta\left(\mathcal{Y}_{j+1}-y_{c m}^{i}(0)\right)\left(t_{\max }-t\right)}\right\rangle \\
& =\langle f(t) f(0)\rangle_{j, t_{\max }-t}
\end{aligned}
$$

where $f^{i}\left(t^{\prime}\right)$ is a function of the complete set of monomer positions of the $i$ th chain, $\left\{\boldsymbol{R}^{n+1}\right\}^{i}$, at time $t^{\prime}$. The Heaviside step function is denoted by $\Theta(y) ; \mathcal{Y}_{j}$ indicates the surface normal position at which the $j$ th region begins: for instance $\mathcal{Y}_{0}$ denotes the position of the surface. The surface normal center of mass position of the $i$ th chain at $t^{\prime}=0$ is $y_{c m}^{i}(0)$. 
In words, eqn 8 is a time average of the time-correlation $f(t) f(0)$, taken with duration $t_{\max }-t$, averaged over all chains whose center of mass lie in the $j$ th region at the beginning of the time average: $t^{\prime}=0$. For brevity, in the following we use the shorthand notation $\langle f(t) f(0)\rangle_{j, t_{\max }-t}$ to represent such a time average, as indicated by the second equality in eqn 8.

The correlation functions were calculated for a total time duration of $t_{\max }=2.4 \tau_{\mathrm{F}}$ for both slip-spring and KG MD models. Over this time period, for both models the center of mass positions of chains in the first two surface regions move a distance normal to the surface of approximately the region width on average. The mean density of chains is similar in every region. We compare the slip-spring model using the parameters: $N=26, N_{s}=0.5$ and $N_{e}^{S S}=4$, with the KG MD model using $n=256$. All the parameters of the slip-spring model have been determined and we keep them fixed throughout, so there is no freedom for any adjustments in all of the following comparison between the slip-spring and MD models.

\section{Chain end-to-end vector correlation function}

Before investigating the dynamical end-to-end vector correlation function we first compare the mean squares of components of the end-to-end vectors of chains in each channel region. The system is invariant with respect to a swap of the surface parallel dimensions $\hat{\boldsymbol{x}}$ and $\hat{\boldsymbol{z}}$. Therefore we average over both dimensions for relevant observables, indicated by the parallel indices, $\|$. Figure 5 plots the mean square end-to-end distance of chains with center of mass position in the $j$ th channel region for both the surface normal and parallel directions $(\alpha=\|, \perp),\left\langle R_{e, \alpha}{ }^{2}\right\rangle_{j}$, normalized by the center channel value, $\left\langle R_{e, \alpha}{ }^{2}\right\rangle_{5}$. The two models agree quite well with regard to this purely static quantity. The mean square of the surface normal component of the end-to-end vector is much smaller in the $j=0$ and $j=1$ regions compared to in the channel center. For the slip-spring model the surface parallel end-to-end distance profile is uniform, whereas for the KG MD model there is a weak swelling in the $j=0$ region which has been discussed previously ${ }^{32}$.

Figure 6 compares normalized time correlation functions of the chain end-to-end vector in the surface parallel direction, $\Phi_{j, \|}(t)$, for chains located in the $j=0$ and $j=5$ (center channel) regions. The normalization is made with respect to the value of the end-to-end

correlation function at $t=0:\left\langle R_{e, \|^{2}}\right\rangle_{j, t_{\max }}$. Note that $\left\langle R_{e, \alpha}{ }^{2}\right\rangle_{j, t_{\max }}$ are dynamical observables 


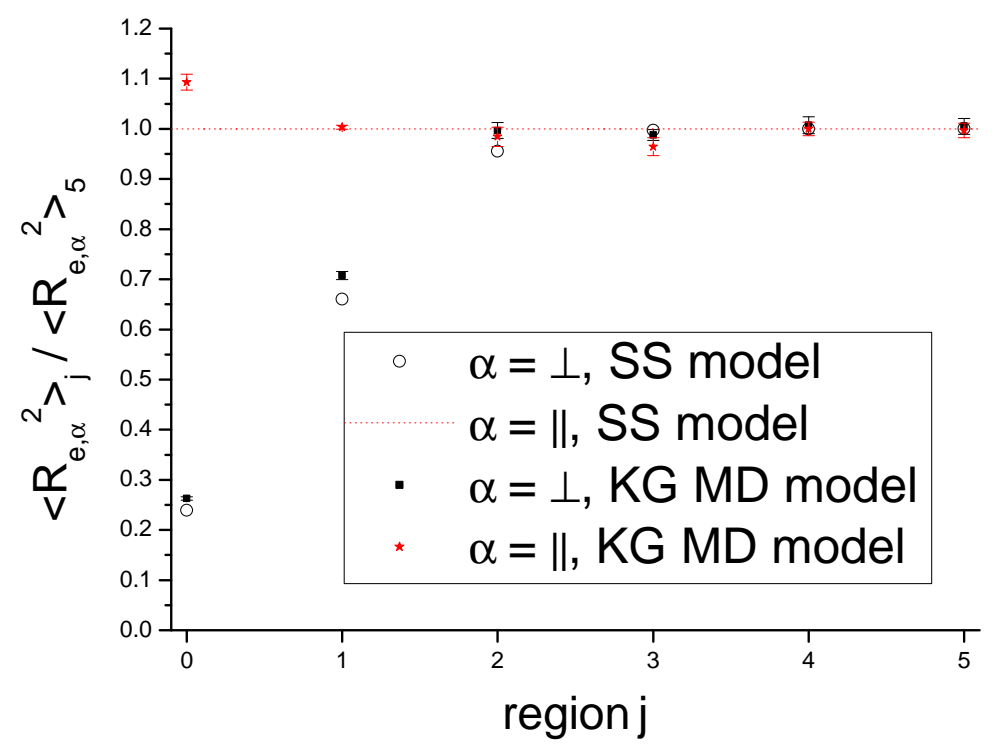

FIG. 5: Mean square end-to-end distances of chains in the $\alpha=\|, \perp$ directions normalized by the center channel value: $\left\langle R_{e, \alpha}{ }^{2}\right\rangle_{j} /\left\langle R_{e, \alpha}{ }^{2}\right\rangle_{5}$. Channel regions are selected depending on the chain center of mass position. Error bars correspond to the KG MD model; error bars are not plotted for the slip-spring model since they are smaller than the size of the symbols.

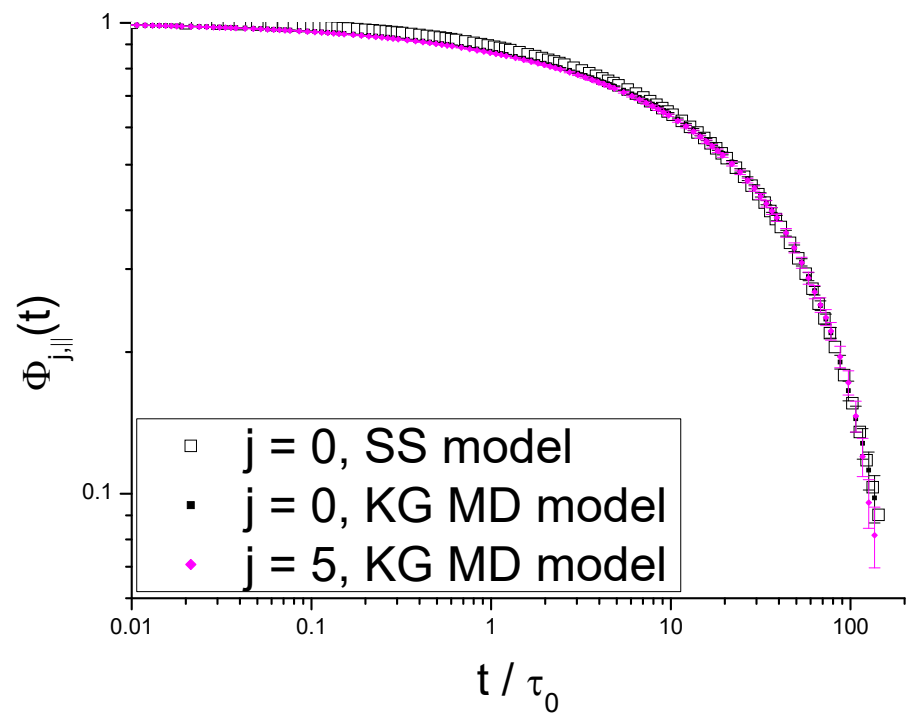

FIG. 6: Normalized end-to-end vector correlation functions in the surface parallel direction, $\Phi_{j, \|}(t)$. The time unit, $\tau_{0}$, is given by eqn 7 , and $\tau_{0} \approx \tau_{e}$. Error bars correspond to the KG MD model. 
that depend on $t_{\max }$. This case corresponds to $f(t)=R_{e, \|}(t) / \sqrt{\left\langle R_{e, \|}^{2}\right\rangle_{j, t_{\max }}}$ in eqn 8. There is no discernible channel dependence for this observable using the slip-spring model which agrees very well with the KG MD model.

Figure 7a plots the end-to-end vector correlation function in the surface normal direction normalized by the center channel value of the end-to-end vector correlation function at $t=0$ : $\left\langle R_{e, \perp}^{2}\right\rangle_{5, t_{\max }}$. This case corresponds to $f(t)=R_{e, \perp}(t) / \sqrt{\left\langle R_{e, \perp}{ }^{2}\right\rangle_{5, t_{\max }}}$ in eqn 8. There is qualitative agreement between the models. Also good quantitative agreement is found up until region $j=1$. But in the $j=0$ region nearest to the surface the slip-spring model does not accurately predict the value of $\left\langle R_{e, \perp}{ }^{2}\right\rangle_{0, t_{\max }} /\left\langle R_{e, \perp}{ }^{2}\right\rangle_{5, t_{\max }}$ that is found using the KG MD model. The disagreement, approximately $20 \%$, is worse than for the purely static

quantity, $\left\langle R_{e, \perp}^{2}\right\rangle_{0} /\left\langle R_{e, \perp}^{2}\right\rangle_{5}$ (see figure 5), for which the disagreement is approximately $10 \%$.

Figure $7 \mathrm{~b}$ plots $\Phi_{j, \perp}(t)$ : the end-to-end vector correlation function in the surface normal direction normalized by $\left\langle R_{e, \perp}{ }^{2}\right\rangle_{j, t_{\max }}$. Figure $7 \mathrm{~b}$ reveals a progressively faster relaxation as the surface is approached. The slip-spring model describes this accelerated relaxation rather accurately except for the region closest to the wall $(j=0)$. In this region there is a notably faster relaxation using the slip-spring model with respect to the KG MD model for this observable.

\section{Mid-monomer mean square displacement}

The mid-monomer mean square displacement in the surface parallel direction is strongly dependent on the choice of surface structure and thermostat friction constant ${ }^{32}$. However this is not the case for the same observable in the surface normal direction, $g_{1, \text { mid, } \perp}(t)$, so that a more meaningful comparison with the slip-spring model can be made for this case. Figure 8 plots $g_{1, \text { mid, } \perp}(t)$ in a normalized form which reveals its sub $\sqrt{t}$ scaling for $\tau_{0} \lesssim t \lesssim \tau_{R}$, where $\tau_{R}$ is an estimated Rouse time for this chain length (see the caption of figure 8). For chains that are not influenced by the surface, this deviation from the $\sqrt{t}$ scaling that is predicted by the Rouse model is associated with topological effects. For $t \gtrsim \tau_{0}$ and $j \geq 1$, the slipspring model matches the KG MD model quite well, and as the surface is approached the mid-monomer diffusion becomes progressively slower using both models. However, using the 


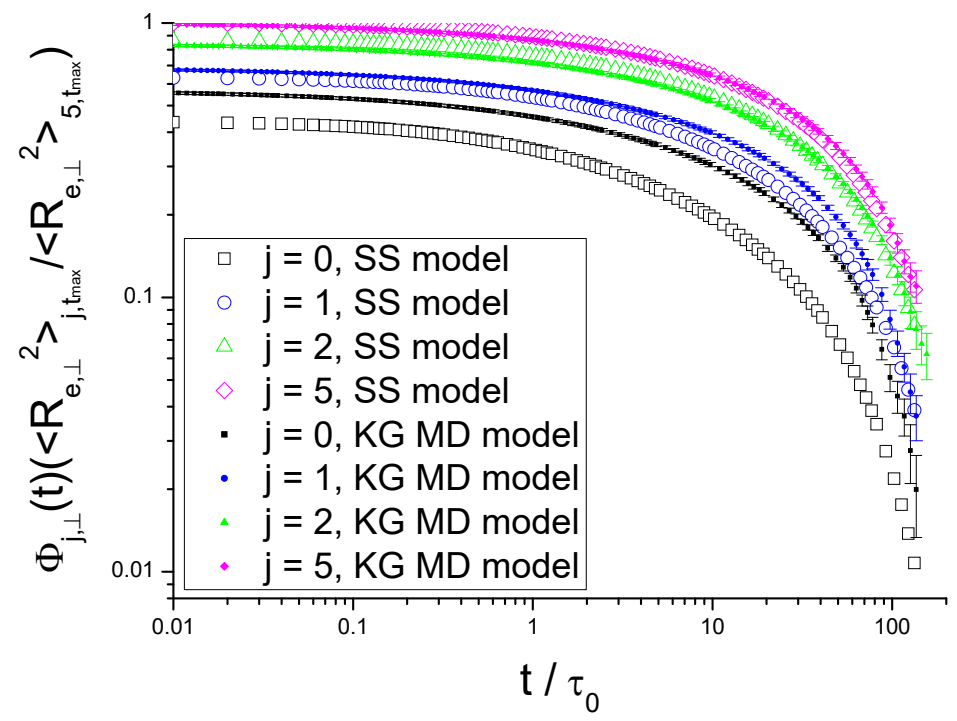

(a)

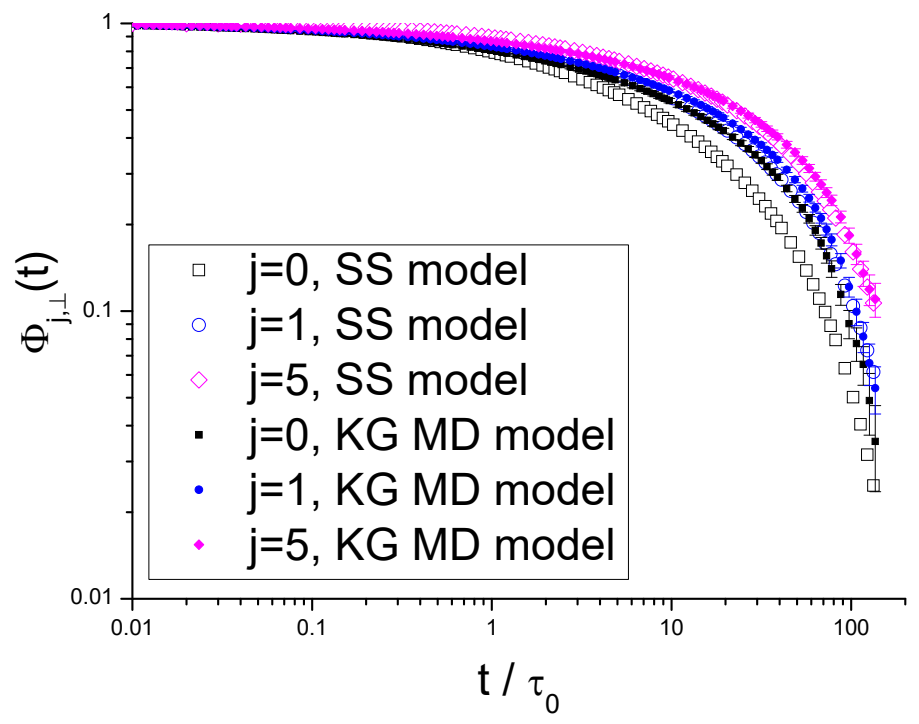

(b)

FIG. 7: Correlation functions of the surface normal component of the end-to-end vector normalized by (a) $\left\langle R_{e, \perp}{ }^{2}\right\rangle_{5, t_{\max }}$ (b) $\left\langle R_{e, \perp}{ }^{2}\right\rangle_{j, t_{\max }}$. Open and filled symbols correspond to the slip-spring and KG MD models respectively. The time unit, $\tau_{0}$, is given by eqn 7 , and $\tau_{0} \approx \tau_{e}$. Error bars correspond to the KG MD model. 


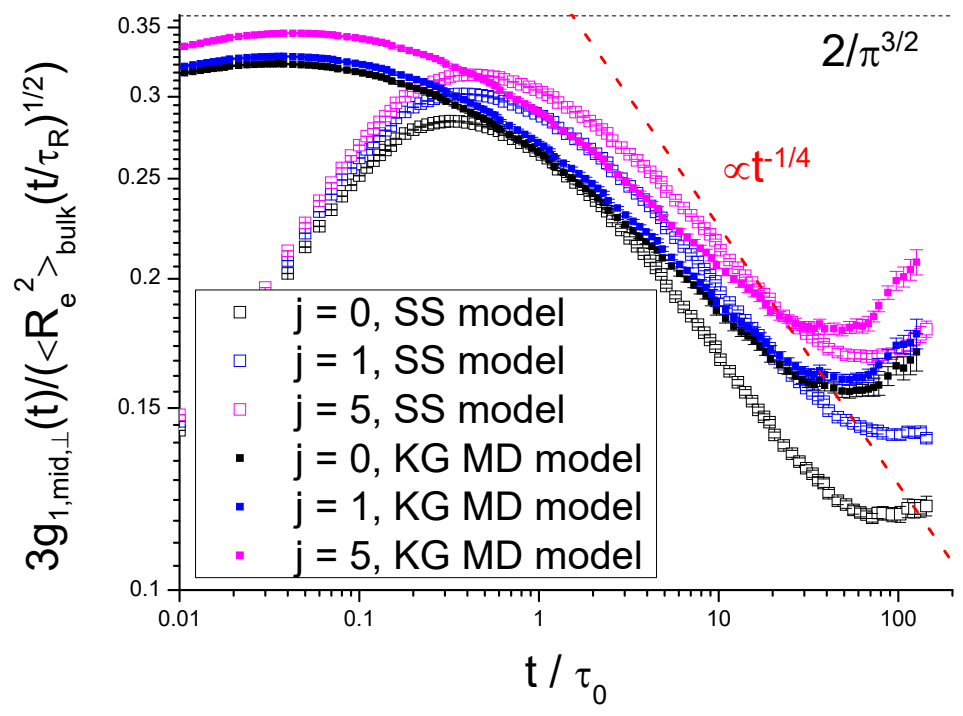

FIG. 8: Mid-monomer mean square displacement in the surface normal direction, normalized to reveal the sub $\sqrt{t}$ time-dependence for $t>\tau_{0}$. The time unit, $\tau_{0}$, is given by eqn 7 , and $\tau_{0} \approx \tau_{e}$. Open and filled symbols correspond to the slip-spring and KG MD models respectively. An estimated Rouse time, $\tau_{R}$, for this chain length is found by scaling from the Rouse time for chains consisting of $n=64$ bonds $^{32}\left(\tau_{R}=23.5 \tau_{0}\right.$ for $\left.n=256\right)$. The black dotted line is $2 / \pi^{3 / 2}$ (continuous limit Rouse model prediction of this observable for $\tau_{N}<t<\tau_{R}$, where $\tau_{N}$ is the relaxation time of the fastest Rouse mode $\left.{ }^{39}\right)$. The red dashed line is proportional to the reptation mode ${ }^{56}$ prediction at $\tau_{e}<t<\tau_{R}$

KG MD model, the mid-monomer diffusion in the $j=0$ region is only very slightly slower than in the $j=1$ region. For the slip-spring model the drop in diffusion rate from the $j=1$ region to the $j=0$ region is considerably larger. Since the KG MD mid-monomers are more mobile than the slip-spring prediction in the immediate proximity of the surface $(j=0)$, this may appear to suggest a decreased degree of entanglement. However the opposite conclusion could be made if we only looked at the surface normal end-to-end vector relaxation, figure 7 . Note also that for $g_{1, \text { mid, } \perp}(t)$ a similar behavior was observed using non-entangled chains ${ }^{32}$ : very close to the surface KG MD chains consisting of $n=64$ bonds have a faster midmonomer diffusion than the (non-entangled) modified-Rouse prediction. Therefore it is not obvious that the speed-up in surface normal diffusion of the MD model with respect to the slip-spring model can be attributed solely to an effect of entanglements. 
Further work is needed to investigate the origin of the wall-mediated effects leading to the discrepancies in the perpendicular relaxation and mid-monomer mean square displacement very close to the surface.

\section{Chain bond orientation correlation function}

A shear component of the chain bond orientation tensor involving the direction normal to the surface is

$$
O_{\| \perp}(t)=\sum_{i=0}^{n-1}\left(r_{i+1, \|}(t)-r_{i, \|}(t)\right)\left(r_{i+1, \perp}(t)-r_{i, \perp}(t)\right)
$$

where $n$ is the number of bonds in a chain. Eqn 9 matches the definition used by Cao and Likhtman $^{37}$. Figure 9a plots the chain bond orientation auto-correlation functions, $\bar{A}_{j}(t)$, which use $f(t)=O_{\| \perp}(t) / n$. This observable is found to be sensitive to the presence of confining walls up to distances of approximately one radius of gyration $(j=0,1)$, while regions further away $(j \geq 2)$ behave very similarly to the center channel $(j=5)$. We observe that the proximity to a confining surface accelerates the relaxation of the bond auto-correlation function for sufficiently long times $\left(t \gtrsim 100 \tau_{\mathrm{LJ}}\right)$. This trend is qualitatively captured by the slip-spring model, although the relaxation in this intermediate time scale is generally overemphasized by the slip-spring model. Nevertheless, for $t \gg \tau_{e}$ the KG MD curves are simultaneously well described by the slip-spring model both near the surface and in the center channel. However, the agreement between the two models is less good for $j=0$ at $t \approx \tau_{e}$ compared to the other two regions. Note that it is not possible to simultaneously fit both the chain mean square end-to-end distance and bond orientation moment, $\bar{A}_{j}(0)$, of a slip-spring model to the KG MD model, even in the bulk. This is due to the effect of chain-swelling, which the ideal chain slip-spring model does not describe ${ }^{32}$. Figure $9 \mathrm{~b}$ compares the ratios $\bar{A}_{0}(t) / \bar{A}_{5}(t)$ and $\bar{A}_{1}(t) / \bar{A}_{5}(t)$, using the slip-spring plots in figure $9 \mathrm{a}$ in addition to plots corresponding to longer chains $(N=104)$ with $N_{s}=0.5$ and $N_{e}^{S S}=4$ held

fixed. For chains nearer the surface, $\bar{A}_{j}(0)$ is smaller. In addition, as discussed above, the decay of correlations is faster near the surface at $t<\tau_{e}$, such that the ratios in figure $9 \mathrm{~b}$ are non-negligibly less than unity for both $j=0$ and $j=1$ regions at $t>\tau_{e}$. The ratio appears to approach 1:1 using the longer slip-spring model chains. This appears unsurprising since in units of the mean bond length the longer chains have a center of mass position further from 


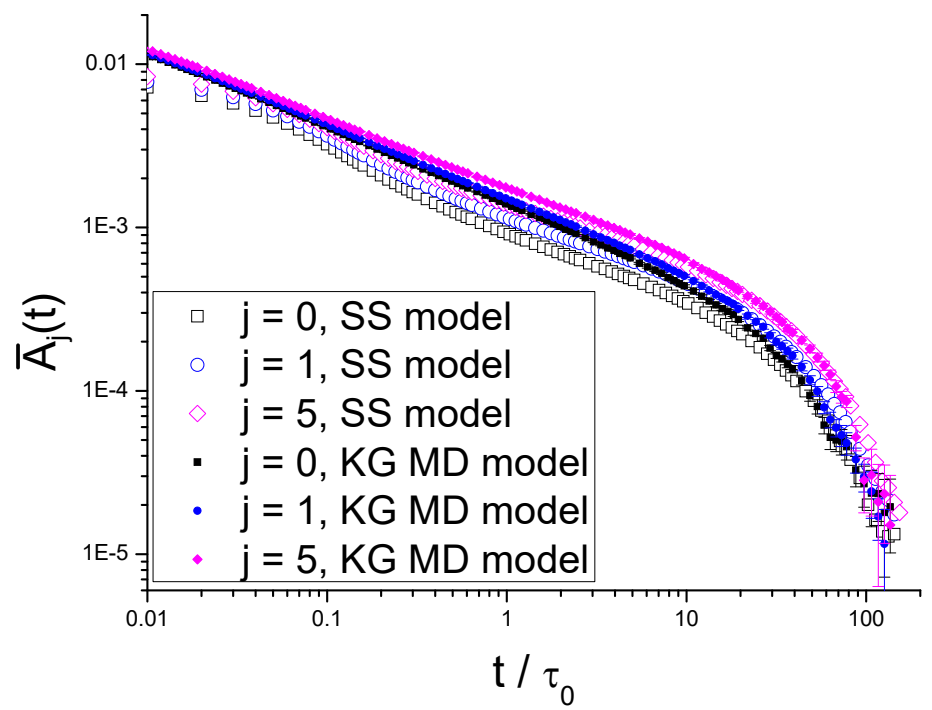

(a)

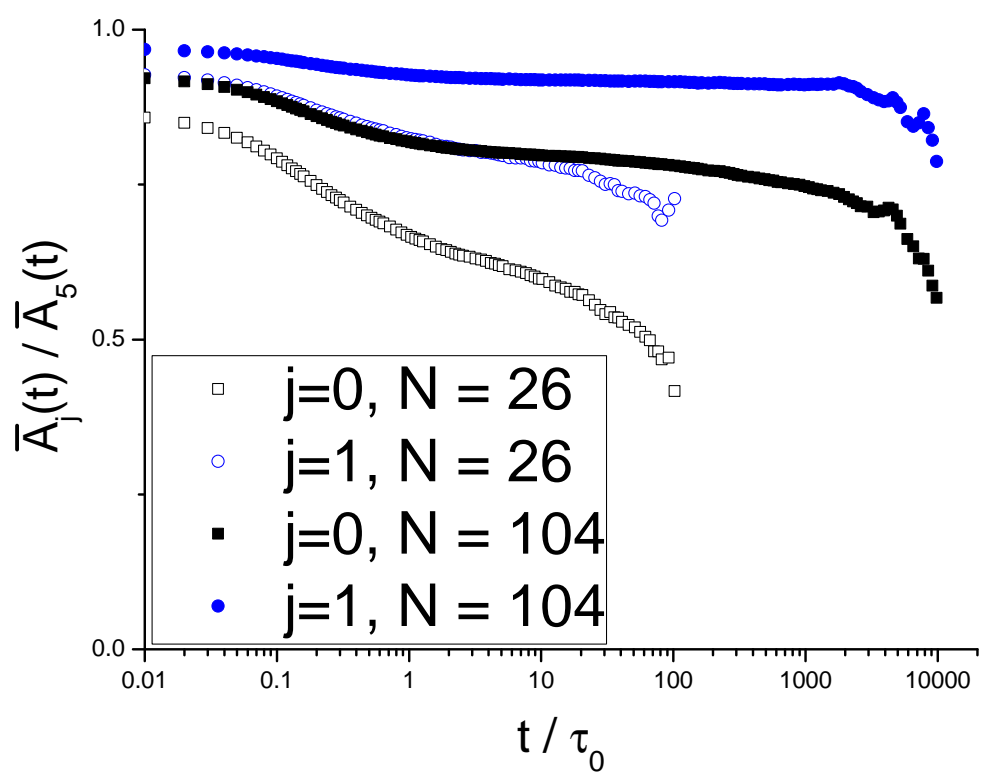

(b)

FIG. 9: (a) Bond orientation correlation functions, $\bar{A}_{j}(t)$. Error bars correspond to the KG MD model. (b) Ratios of the bond orientation correlation functions using the slip-spring model, $N_{e}=4$ and $N_{s}=0.5$. Black: $\bar{A}_{0}(t) / \bar{A}_{5}(t)$; blue: $\bar{A}_{1}(t) / \bar{A}_{5}(t)$. Open symbols use $N=26$. Filled symbols use $N=104$. The time unit, $\tau_{0}$, is given by eqn 7 , and $\tau_{0} \approx \tau_{e}$ 
the surface compared to the shorter chains, and will correspondingly have a lower proportion of bonds that are near the surface.

The plateau modulus is often defined theoretically ${ }^{56}$ as the value of the linear shear stress relaxation function at $\tau_{e}: G\left(\tau_{e}\right)$ (also $\sec ^{57}$ for a discussion of the relation between this theoretical definition and experimental definitions of the plateau modulus). The Stress Optical Rule (SOR) can be used to relate $G(t)$ to the total system bond shear orientation correlation function, $S(t)$, which includes cross-chain bond correlations ${ }^{37,58} . S(t)$ is defined (following the notation of Cao and Likhtman ${ }^{37}$ ) as

$$
\begin{aligned}
S(t) & =\frac{N_{b}}{T} \sum_{l=1}^{N_{c h}} \sum_{m=1}^{N_{c h}} \frac{1}{N_{b}^{2}}\left\langle O_{\| \perp}^{l}(t) O_{\| \perp}^{m}(0)\right\rangle \\
& =A(t)+C(t)
\end{aligned}
$$

where $N_{b}=N_{c h} n$ is the total number of bonds in the system, and $A(t)$ and $C(t)$ are the auto-chain and cross-chain contributions to $S(t)$

$$
\begin{aligned}
A(t) & =\frac{N_{b}}{T} \sum_{l=1}^{N_{c h}} \frac{1}{N_{b}^{2}}\left\langle O_{\| \perp}^{l}(t) O_{\| \perp}^{l}(0)\right\rangle=\frac{N_{b}}{T N_{\text {reg }}} \sum_{j=0}^{N_{\mathrm{reg}}-1} \bar{A}_{j}(t) \\
C(t) & =\frac{N_{b}}{T} \sum_{l=1}^{N_{c h}} \sum_{m=1, m \neq l}^{N_{c h}} \frac{1}{N_{b}^{2}}\left\langle O_{\| \perp}^{l}(t) O_{\| \perp}^{m}(0)\right\rangle
\end{aligned}
$$

where $N_{\text {reg }}$ is the total number of distinct regions comprising the fluid (in our case $N_{\text {reg }}=6$ ). The SOR states that $G(t) \propto S(t)$. For bulk systems it has been demonstrated ${ }^{37}$ that at around $\tau_{e}$ the cross-chain and auto-chain contributions have a similar magnitude (and form), $C\left(\tau_{e}\right) \approx A\left(\tau_{e}\right)$. This is true for a variety of blends of linear chains ${ }^{37}$ such that $G\left(\tau_{e}\right)$ becomes a function of $A\left(\tau_{e}\right)$, and we may write $G\left(\tau_{e}\right)=\beta A\left(\tau_{e}\right)$ with some generality, where $\beta$ is constant for a range of blend composition. However the behavior of cross-chain correlations in confinement could in principle be different from a corresponding bulk system: see e.g. Cho et $\mathrm{al}^{59}$. A preliminary investigation ${ }^{36}$ into cross-chain correlations in non-entangled and confined linear melts reveals non-trivial behavior that depends upon the surface structure and whether there are surface adsorbed chains present. For the simplified case of nonadsorbing roughened walls consisting of Lennard-Jones particles, the relative importance of cross-correlations was found to be similar to the bulk case. If the SOR is valid for a confined polymer melt and we observe the same relationship: $A_{\text {confined }}\left(\tau_{e}\right) \approx C_{\text {confined }}\left(\tau_{e}\right)$, as for bulk melts, the relation $G\left(\tau_{e}\right)=\beta A\left(\tau_{e}\right)$ may be further generalized to confined systems. Figure 9 
would then suggest that the plateau modulus may be lower for a confined system compared to a bulk melt. It may then appear plausible that the prediction of figure $9 \mathrm{~b}$ could explain part of the drop in the plateau modulus observed by Weir et al. ${ }^{11}$. Note however that this analysis doesn't take into account the influence of surface adsorbed chains that may have slower segmental relaxation ${ }^{32}$ at $t<\tau_{e}$ which could actually increase the plateau modulus. If $N_{e}^{\text {topo }}$ is unchanged in confinement then the relationship of the plateau modulus with $N_{e}^{\text {topo }}$ may be different from a corresponding bulk relation.

It has previously been demonstrated ${ }^{32}$ that the zeroth Rouse mode (center of mass) motion of KG MD model chains is quite highly dependent upon the value of the thermostat friction used. This is due to the importance of momentum conservation for Hydrodynamic Interactions $(\mathrm{HI})^{60}$. Following further investigation ${ }^{36}$ we have found that the center of mass diffusion coefficient in a direction parallel to the surface also depends strongly on surface structure (whether the surface reflects momentum: flat reflective, or not: roughened lattice surface), even beyond the Rouse time, using short chains comprising $n=64$ bonds. As a result, it may not be straightforward or necessarily meaningful to compare zeroth mode motion in slip-spring model simulations to that of the KG MD model, since the HI and surface structure effects are not described by the single-chain model. In contrast, higher mode autochain correlation functions (i.e. not dependent on cross-chain correlations) are very weakly, if at all, dependent on the boundary structure or thermostat friction. Therefore it is natural that we first investigated the behavior of observables that depend only on non-zero Rouse modes, such as the chain end-to-end vector and bond orientation auto-correlation functions, and compared the results to KG MD using a flat reflective surface and a Langevin thermostat with the most commonly used friction constant in the literature that allows for a large time-step.

\section{CONCLUSION}

We have investigated a KG MD model of a polymer melt weakly confined in a channel with flat reflective boundaries. No discernible confinement effect was found in the terminal relaxation time of the end-to-end vector correlation function in the surface parallel direction for chains with different center of mass positions relative to a surface. Despite this we find that the auto-chain bond shear orientation correlation functions for chains in different re- 
gions depend on the distance of the chains to the confining wall by a non-negligible amount at $t>\tau_{e}$. We proposed a minimal and parameter-free extension of a well-known slip-spring model $^{43}$ to include confinement effects via the modified-Rouse model proposed by two of us recently ${ }^{32}$. For simplicity, we assume a uniform density of slip-springs in the channel. Despite its simplicity, at $t \gg \tau_{e}$, both the bond orientation and the normalized surface parallel end-to-end vector correlation functions were simultaneously quite well described by the single-chain model. However the single-chain model agreed less well with the MD simulation for the surface normal end-to-end vector correlation function and mid-monomer mean square displacement, $\Phi_{j, \perp}(t)$ and $g_{1, \text { mid, } \perp}(t)$ respectively. Modifications of the proposed confined slip-spring model for an improved comparison of $\Phi_{0, \perp}(t)$ and $g_{1, \text { mid, } \perp}(t)$ is left for future work.

Our simulation results do not support the picture of strong disentanglement at flat surfaces in weak confinement that has sometimes been both theoretically suggested ${ }^{17}$, and experimentally inferred ${ }^{18}$ in the past. The reasonably good agreement between the MD model and slip-spring model suggests that further such comparison using longer chain lengths could be fruitful, and may be used to provide a more precise measure of any disentanglement (or lack there of) at the flat surface.

The behavior of the auto-chain bond orientation correlation function, using the slip-spring model with a uniform density of slip-links in the channel, suggests that the experimentally observed plateau modulus could be different in confined melts even when there is no drop in topological interactions.

In the future it would be desirable to compare our results with multi-chain slip-spring models which could, in principle, also use a uniform density of slip-springs within the channel. In many multi-chain models ${ }^{61-64}$ slips-springs are qualitatively different from those in the singlechain model, since they are explicit binary interactions acting between two different chains. It is feasible that different dynamical behavior may be observed for such cases. An existing confined formulation ${ }^{54}$ of such a multi-chain slip-spring model has a depletion of slip-springs at the walls, and the mean number of slip-springs per chain monotonically decreases as the channel width is reduced. A Primitive Chain Network model ${ }^{65}$ has qualitatively similar behavior in this respect. For the model used in the current work, if the channel is narrowed a uniform slip-spring density profile is maintained, and reflective-random walk statistics are also conserved, providing that the channel width greatly exceeds the characteristic length 
scales of the virtual and real spring potentials: $L_{c} \gg \sqrt{N_{s}} b$ and $L_{c} \gg b$ respectively. However, for channel widths much smaller than the bulk chain dimension $\left(L_{c} \ll \sqrt{N} b\right)$ this behavior is expected to become considerably less realistic since disentanglement and a greater degree of chain swelling are eventually expected ${ }^{9,66}$. Generally if the parameters of a coarse-grained model are changed (e.g. reducing the channel width), it should be verified that the model remains consistent with experiment and/or detailed simulation.

\section{SUPPLEMENTARY MATERIAL}

See the supplementary material for details and discussion of the slip-spring lifetime distributions as a function of the slip-spring position in the channel when constraint release is switched off.

\section{ACKNOWLEDGMENTS}

We thank Professor Martin Kröger for providing the Z1 algorithm for our use, and Professors Jean-Louis Barrat and Marcus Müller for useful discussion.

The essential research question considered in this work was originally proposed by Professor Alexei Likhtman: what is an entanglement at the surface?

This work was supported by the Engineering and Physical Sciences Research Council (EPSRC), grant ref: EP/K503125/1. P. Ilg was also supported by a grant from the European Union, FP7-MC-CIG Grant No. 631233. Z. Wang is thankful for the support from the Engineering and Physical Sciences Research Council (EPSRC) Grants No. EP/L020599/1.

\section{REFERENCES}

${ }^{1}$ G. Allegra, G. Raos, and M. Vacatello, Progr. Polym. Sci. 33, 683 (2008).

${ }^{2}$ S. S. Sternstein and A.-J. Zhu, Macromolecules 35, 7262 (2002).

${ }^{3}$ S. G. Hatzikiriakos, Progr. Polym. Sci. 37, 624 (2012).

${ }^{4}$ L. Léger, H. Hervet, G. Massey, and E. Durliat, J. Phys: Cond. Mat. 9, 7719 (1997).

${ }^{5}$ C. McIlroy and P. D. Olmsted, Polymer 123, 376 (2017).

${ }^{6}$ W. Bisbee, J. Qin, and S. T. Milner, Macromolecules 44, 8972 (2011).

${ }^{7}$ M. Vladkov and J.-L. Barrat, Macromolecules 40, 3797 (2007). 
${ }^{8}$ D. M. Sussman, W.-S. Tung, K. I. Winey, K. S. Schweizer, and R. A. Riggleman, Macromolecules 47, 6462 (2014).

${ }^{9}$ N.-K. Lee, D. Diddens, H. Meyer, and A. Johner, Phys. Rev. Lett. 17, 067802(5) (2017).

${ }^{10}$ H. Bodiguel and C. Fretigny, Macromolecules 40, 7291 (2007).

${ }^{11}$ M. P. Weir, D. W. Johnson, S. C. Boothroyd, R. C. Savage, R. L. Thompson, S. M. King, S. E. Rogers, K. S. Coleman, and N. Clarke, ACS Macro. Lett. 5, 430 (2016).

${ }^{12}$ A. Karatrantos, R. J. Composto, K. I. Winey, M. Kröger, and N. Clarke, Macromolecules 45, $7274(2012)$.

${ }^{13}$ L. J. Fetters, D. J. Lohse, D. Richter, T. A. Witten, and A. Zirkel, Macromolecules 27, 4639 (1994).

${ }^{14}$ S. T. Milner, Macromolecules 38, 4929 (2005).

${ }^{15}$ Y.-H. Lin, Macromolecules 20, 3080 (1987).

${ }^{16}$ T. A. Kavassalis and J. Noolandi, Phys. Rev. Lett. 59, 2674 (1987).

${ }^{17}$ H. R. Brown and T. Russell, Macromolecules 29, 798 (1996).

${ }^{18}$ O. Bäumchen, R. Fetzer, and K. Jacobs, Phys. Rev. Letts. 103, 247801 (2009).

${ }^{19}$ R. Everaers, S. K. Sukumaran, G. S. Grest, C. Svaneborg, A. Sivasubramanian, and K. Kremer, Science 303, 823 (2004).

${ }^{20}$ R. S. Hoy, K. Foteinopoulou, and M. Kröger, Phys. Rev. E. 80, 031803 (2009).

${ }^{21}$ S. Shanbhag and M. Kröger, Macromolecules 40, 2897 (2007).

${ }^{22}$ A. Karatrantos, N. Clarke, R. J. Composto, and K. I. Winey, Soft Matter 9, 3877 (2013).

${ }^{23}$ A. Karatrantos, N. Clarke, R. J. Composto, and K. I. Winey, Soft Matter 12, 2567 (2016).

${ }^{24}$ D. M. Sussman, Phys. Rev. E. 94, 012503 (2016).

${ }^{25}$ R. Everaers, Phys. Rev. E. 86, 022801 (2012).

${ }^{26}$ A. Likhtman, Soft Matter 10, 1895 (2014).

${ }^{27}$ J. Cao and Z. Wang, Macromolecules 49, 5677 (2016).

${ }^{28}$ J. Cao, J. Qin, and S. T. Milner, Macromolecules 47, 2479 (2014).

${ }^{29}$ S. K. Sukumaran and A. E. Likhtman, Macromolecules 42, 4300 (2009).

${ }^{30}$ Z. Wang, A. E. Likhtman, and R. G. Larson, Macromolecules 45, 3557 (2012).

${ }^{31}$ A. Ramírez-Hernández, B. L. Peters, L. Schneider, M. Andreev, J. D. Schieber, M. Müller, M. Kröger, and J. J. de Pablo, Macromolecules 51, 2110 (2018).

${ }^{32}$ J. Kirk and P. Ilg, Macromolecules 50, 3703 (2017).

${ }^{33}$ K. Kremer and G. S. Grest, J. Chem. Phys. 92, 5057 (1990). 
${ }^{34}$ J. Baschnagel and F. Varnik, J. Phys.: Cond. Mat. 17, R851 (2005).

${ }^{35}$ B. C. Abberton, W. K. Liu, and S. Keten, Macromolecules 48, 7631 (2015).

${ }^{36} \mathrm{~J}$. Kirk, The molecular dynamics and rheology of polymer melts near the flat surface, Ph.D. thesis, School of Mathematical, Physical and Computational Sciences, University of Reading (2018), https://www.reading.ac.uk/web/files/maths/Kirk-thesis.pdf.

${ }^{37}$ J. Cao and A. E. Likhtman, Phys. Rev. Lett. 104, 207801 (2010).

${ }^{38}$ J. Cao and A. E. Likhtman, ACS Macro. Lett. 4, 1376 (2015).

${ }^{39}$ A. E. Likhtman, in Polymer Science: A Comprehensive Reference, Vol. 10, edited by K. Matyjaszewski and M. Müller (Elsevier B.V., 2012) p. 133.

${ }^{40}$ J. Cao, J. Qin, and S. T. Milner, Macromolecules 48, 99 (2015).

${ }^{41}$ Thanks to Jean-Louis Barrat for pointing this out.

${ }^{42}$ S. K. Sukumaran, G. S. Grest, K. Kremer, and R. Everaers, J. Pol. Sci. B: Pol. Phys. 43, $917(2005)$.

${ }^{43}$ A. E. Likhtman, Macromolecules 38, 6128 (2005).

${ }^{44}$ A. Silberberg, J. Colloid Int. Sci. 90, 86 (1981).

${ }^{45}$ E. Masnada, S. Merabia, M. Couty, and J.-L. Barrat, Soft Matter 9, 10532 (2013).

${ }^{46}$ J. Zhu, A. E. Likhtman, and Z. Wang, J. Chem. Phys. 147, 0044907 (2017).

${ }^{47}$ J. Zhu, Multiscale Computer Simulation Studies of Entangled Branched Polymers, Ph.D. thesis, Department of Mathematics and Statistics, University of Reading (2016).

${ }^{48}$ K. C. Daoulas, V. A. Harmandaris, and V. G. Mavrantzas, Macromolecules 38, 5780 (2005).

${ }^{49}$ V. A. Harmandaris, V. G. Mavrantzas, D. N. Theodorou, M. Kröger, J. Ramirez, H. C. Öttinger, and D. Vlassopoulos, Macromolecules 36, 1376 (2003).

${ }^{50}$ S. Lee, A. V. Lyulin, C. W. Frank, and D. Y. Yoon, Polymer 116, 540 (2017).

${ }^{51}$ P. Gutfreund, O. Bäumchen, R. Fetzer, D. Grinten, M. Maccarini, K. Jacobs, H. Zabel, and M. Wolff, Phys. Rev. E 87, 012306 (2013).

${ }^{52}$ J. R. Howse, R. Steitz, M. Pannek, P. Simon, D. W. Schubert, and G. H. Findenegg, Phys. Chem. Chem. Phys. 3, 4044 (2001).

${ }^{53}$ M. Müller, B. Steinmüller, K. C. Daoulas, A. Ramírez-Hernández, and J. J. de Pablo, Phys. Chem. Chem. Phys. 13, 10491 (2011).

${ }^{54}$ A. Ramírez-Hernández, B. L. Peters, L. Schneider, M. Andreev, and J. D. Schieber, J. Chem. Phys. 146, 014903 (2017). 
${ }^{55}$ M. Rubinstein and R. H. Colby, Polymer Physics (Oxford University Press, Oxford, UK, 2003).

${ }^{56}$ M. Doi and S. F. Edwards, The Theory of Polymer Dynamics (Clarendon Press, 1986).

${ }^{57}$ A. E. Likhtman and T. C. B. McLeish, Macromolecules 35, 6332 (2002).

${ }^{58}$ Y. Masubuchi and S. Sukumaran, J. Soc. Rheol. 41 (2013).

${ }^{59}$ Y.-K. Cho, H. Watanabe, and S. Granick, J. Chem. Phys. 110, 9688 (1999).

${ }^{60}$ J. Farago, H. Meyer, J. Baschnagel., and A. N. Semenov, Phys. Rev. E. 85, 051807 (2012).

${ }^{61}$ T. Uneyama and Y. Masubuchi, J. Chem. Phys. 137, 154902 (2012).

${ }^{62}$ V. C. Chappa, D. C. Morse, A. Zippelius, and M. Müller, Phys. Rev. Lett. 109, 148302 (2012).

${ }^{63}$ A. Ramírez-Hernández, F. A. Detcheverry, B. L. Peters, V. C. Chappa, K. S. Schweizer, M. Müller, and J. J. de Pablo, Macromolecules 46, 6287 (2013).

${ }^{64}$ M. Langeloth, Y. Masubuchi, M. C. Böhm, and F. Müller-Plathe, J. Chem. Phys. 138, 104907 (2013).

${ }^{65}$ S. Okuda, Y. Inoue, Y. Masubuchi, T. Uneyama, and M. Hojo, J. Chem. Phys. 130, 214907 (2009).

${ }^{66}$ N. A. Garcia and J.-L. Barrat, Macromolecules 51 (2018). 